\title{
MRI evaluation of tibial tunnel wall cortical bone formation after platelet-rich plasma applied during anterior cruciate ligament reconstruction
}

\author{
Mitja Rupreht ${ }^{1}$, Matjaž Vogrin², Mohsen Hussein ${ }^{3}$ \\ ${ }^{1}$ Radiology Department, University Medical Center Maribor, Medical Faculty, University of Maribor, Slovenia \\ ${ }^{2}$ Orthopaedics Department, University Medical Center Maribor, Medical Faculty, University of Maribor, Slovenia \\ ${ }^{3}$ Artros Center for Orthopaedic Surgery and Sports Medicine, Ljubljana, Slovenia
}

Radiol Oncol 2013; 47(2): 119-124.

Received 29 May 2012

Accepted 6 September 2012

Correspondence to: Mitja Rupreht, MD, PhD, Radiology Department, University Medical Center Maribor, Medical Faculty, University of Maribor, Slovenia. Phone: +386 (0)2 3212 255; Fax: +386 (0)2 3212 982; E-mail: mitja.rupreht@guest.arnes.si

Disclosure: No potential conflicts of interest were disclosed.

Background. After anterior cruciate ligament $(\mathrm{ACL})$ reconstruction, formation of cortical sclerotic bone encircling the femoral and tibial tunnel is a part of intratunnel graft healing. During the physiological cascades of soft tissue healing and bone growth, cellular and hormonal factors play an important role. The purpose of this study was to noninvasively but quantitatively assess the effect of intraoperatively applied platelet-rich plasma (PRP) on the formation of cortical bone encircling the tibial tunnel.

Patients and methods. In fifty patients, standard arthroscopic ACL reconstructions were performed. The PRP group $(n=25)$ received a local application of PRP while the control group $(n=25)$ did not receive PRP. The proximal tibial tunnel was examined by MRI in the paraxial plane where the portion of the tibial tunnel wall circumference consisting of sclerotic cortical bone was assessed with testing occurring at one, two and a half and six months after surgery. Results. At one month after surgery, differences between the groups in the amount of cortical sclerotic bone encircling the tunnel were not significant ( $p=0.928)$. At two and a half months, the sclerotic portion of the tunnel wall in the PRP group (36.2\%) was significantly larger than in the control $(22.5 \%)$ group $(p=0.004)$. At six months, the portion of sclerotic bone in the PRP group (67.1\%) was also significantly larger than in the control (53.5\%) group ( $p=0.003)$. Conclusions. Enhanced cortical bone formation encircling the tibial tunnel at 2.5 and 6 months after ACL graft reconstruction results from locally applied platelet-rich plasma.

Key words: anterior cruciate ligament graft; platelet-rich plasma; tibial tunnel; cortical bone; MRI

\section{Introduction}

After anterior cruciate ligament (ACL) reconstruction, two biological mechanisms take place: ligamentization of the intra-articular part of the graft and healing in the bone tunnel. ${ }^{1-5}$

Among other processes, in the chronic reparative phase of intratunnel graft healing, chondrification, neo-ossification and proliferative osteoblastic activity are present at about 6 weeks after recon- struction $^{3}$ with the formation of new cortical bone, creating the tunnel wall.

During the physiological cascades of soft tissue healing and bone growth, cellular and hormonal factors play an important role, the most important among them being various growth factors (GF).5,6 These proteins have a positive effect on fibroblast proliferation and the synthesis of extracellular matrix proteins and therefore on the enhancement of tissue healing. ${ }^{7-9}$ Platelet-derived growth factors 
(PDGFs) particularly enhance the graft incorporation process. ${ }^{9}$

Platelet-rich plasma (PRP), defined as a portion of the plasma fraction of autologous blood having a platelet concentration above the baseline, contains an autologous concentration of platelets and growth factors. ${ }^{9,10}$ PRP can be activated with thrombin to create platelet-rich plasma gel. The role of local application of various GF in ACL reconstruction has been analysed by previous studies in animals, where their effect has been evaluated with histological findings or biomechanical tests. ${ }^{11-14}$

MRI is because of its excellent contrast resolution $^{15,16}$ feasible method for demonstrating the osteosclerosis, where cortical bone is hypointense on all pulse sequences. To the best of our knowledge, no radiological research has yet evaluated the effect of PRP on the formation of cortical bone encircling the tibial tunnel after ACL reconstruction in humans. We found only one study, which assessed the cortication of the tunnel wall, however, only its presence was evaluated with $\mathrm{CT}$, in comparing two different graft fixation screws. ${ }^{17}$ The enhancing effect of PRP and bone GF on bone formation was demonstrated by histological studies in animal models as well as in periodontology. ${ }^{18-25}$

Therefore, the aim of presented study was to assess the effect of intraoperatively locally applied PRP, using MRI, for quantitative but noninvasive measurement of the cortical sclerotic bone formation, encircling the tibial tunnel. We hypothesized that PRP promotes the tunnel wall cortical bone (TCB) formation that can be quantitatively assessed by MRI.

\section{Patients and methods}

\section{Patient selection, surgical technique and platelet gel preparation}

The study was designed as a 6-month, single-centre trial, approved by the national ethics committee and was carried out in accordance with the ethical standards laid down in the 1964 Declaration of Helsinki. All patients gave their written informed consent for participation in the study.

The 50 patients included in the study, aged between 18 and 50, were treated for ACL rupture. The main indication for reconstruction was a symptomatic, unstable knee joint due to ACL rupture, assessed by the orthopaedic surgeon. All patients with inflammatory diseases, diabetes mellitus, advanced knee osteoarthrosis ( $3^{\text {rd }}$ and $4^{\text {rd }}$ degree), previous knee surgery (osteotomies, reconstructive ligament and meniscal procedures and chondral lesions treatment), malignant diseases, allergy to the contrast media, renal diseases and thrombocytopenia were excluded from the study.

All patients undergoing arthroscopic ACL reconstruction were randomized into 2 groups: PRP group comprised 25 patients ( 15 men, 10 women), and the control group (16 men, 9 women).

All procedures were performed by the same orthopaedic surgeon. In all cases, the standard arthroscopic reconstructive procedure, using the single-incision technique with a double-looped semitendinosus and gracilis tendon graft, was performed. The tunnel sizes in the tibia and femur were matched to the cross-sectional size of the graft and measured 7-9 $\mathrm{mm}$ in diameter. The graft was inserted antegrade via the tibial and femoral tunnel and fixed with 2 bioabsorbable cross pins $(3.2 \mathrm{~mm}$, DePuy Mitek, Massachussets, USA) in the femoral tunnel and with one bioabsorbable interference screw (8-10 mm, DePuy Mitek, Massachusetts, USA) in the tibial tunnel.

The patients in the platelet group received a local application of PRP, which the patients in the control group did not receive. PRP preparation was similar to that previously described. ${ }^{26,27}$ During the surgical procedure; autologous blood was obtained and centrifuged. The fraction of PRP was then mixed with activated autologous human thrombin and applied after autograft positioning, into the femoral and tibial tunnels $(1 \mathrm{ml}$ in each of them), as well as onto the graft itself $(3 \mathrm{ml})$, where the autologous PRP was formed. An interference screw was inserted after PRP application. All patients were blinded to the treatment with PRP. Both groups followed the same standard rehabilitation protocol.

\section{Radiological assessment}

The MRI was performed in proton density (PD) sequence with fat suppression (TR 2900ms, TE $22 \mathrm{ms,}$ 3 NEX, matrix 320×224, FOV 200, slice thickness 3 $\mathrm{mm}, 1 \mathrm{~mm}$ spacing). The tibial tunnel was examined in the paraxial plane, perpendicular to the tunnel axis. The tibial tunnel is more easily identified than the femoral tunnel on scout images, which facilitates planning and analysis. Examinations were performed one, two and a half and six months after the ACL reconstruction.

At the first examination one month after reconstruction, the slice with the most pronounced TCB between the tibial plateau and the tip of the inter- 

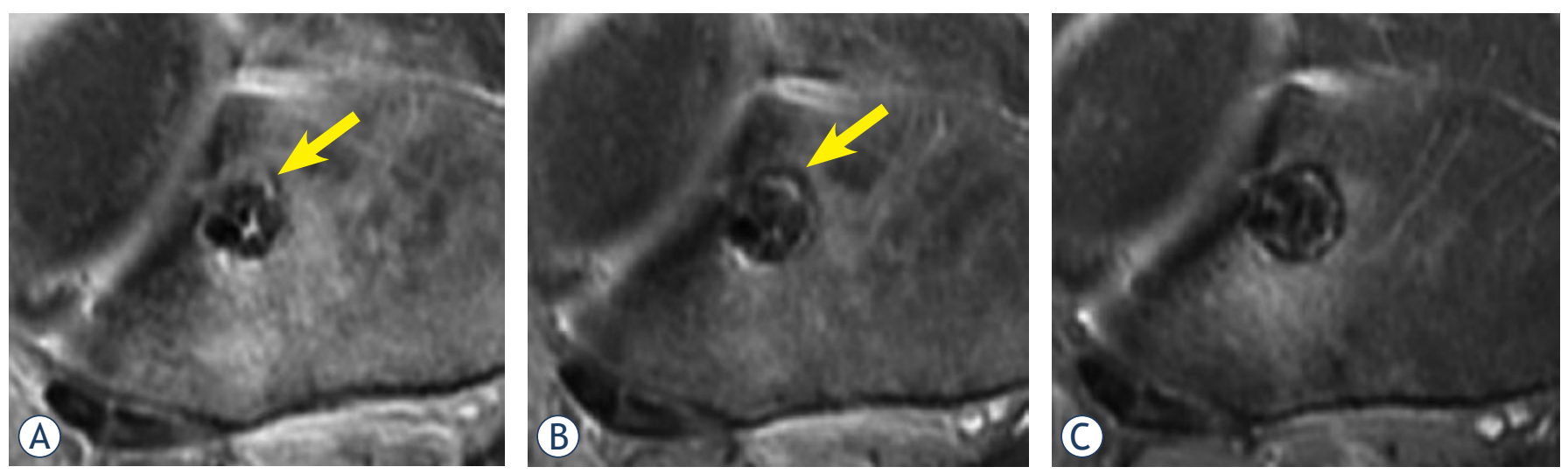

FIGURE 1. Proton-density weighted fat-suppressed paraxial images just below the tibial plateau from the same patient one (A), two and a half (B) and six (C) months after reconstruction. Because of the perpendicular orientation of slices the cross section of the tibial tunnel was in the rule circular. At the first month (A), only small part of the tunnel wall is sclerotic (estimated to be $10 \%$, arrow). At two and a half months (B) about $20 \%$ of the tunnel wall is sclerotic. At six months (C), a thick sclerotic rim encircles estimated $90 \%$ of the tunnel. Note also some high signal intensity surrounding the tunnel, representing oedema, which also decreased during the follow-up.

ference screw, where images were free of volume averaging from the plateau and artefacts from the screw, was chosen for the analysis. In each patient, the same slice was chosen in follow-up assessments.

TCB was defined as a clearly hypointense rim of the tibial tunnel wall that was at least $1 \mathrm{~mm}$ thick, assessed on paraxial slices. The portion of the tunnel wall circumference, consisting of TCB, was assessed by the consensus of the radiologist (M.R.) and the orthopaedic surgeon (M.V.), rounded off to ten percent (Figure 1). The examiners were blinded to group assignment but not to the time of the procedure.

\section{Statistical analysis}

Numerical data are presented as mean values, while categorical data are expressed as proportions. Differences in TCB between the platelet and control groups were analysed using the MannWhitney test. Changes in TCB from the first to the final examination after ACL reconstruction were analysed by the Friedman two-way analysis of variance. As the post-hoc tests, Wilcoxon's signed ranks tests with Keppels modification of the Bonferroni correction of alpha were used. A P value less than 0.05 were taken to represent statistical significance. Data were analysed using PASW 18 software (SPSS Inc., Chicago, IL, USA).

\section{Results}

20 patients from the control group and 21 patients from the PRP group were available for the followup and were analysed, while 9 patients from initial group were lost to follow-up. A comparison of the preoperative parameters of remaining patients in the PRP and the control groups showed that both groups were comparable in gender, age, injury site and body mass index (Table 1).

We observed a gradual increase in the percentage of the tunnel wall consisting of TCB during the follow-up (Table 2). In each group, post-hoc comparisons showed a significant increase in average

TABLE 1. Preoperative data on injured patients

\begin{tabular}{|c|c|c|c|}
\hline Characteristic & $\begin{array}{l}\text { Control group } \\
(n=20)\end{array}$ & $\begin{array}{l}\text { PRP group } \\
\quad(n=21)\end{array}$ & $\begin{array}{l}\text { p-value for differences } \\
\text { between groups }\end{array}$ \\
\hline Men, n (\%) & $15(75)$ & $13(62)$ & 0.505 \\
\hline Age, years & $32.6 \pm 12.3$ & $37.2 \pm 8.4$ & 0.112 \\
\hline Injured knee, n (\%) & & & 1.000 \\
\hline Right & $12(60 \%)$ & $12(57 \%)$ & \\
\hline Left & $8(40 \%)$ & 9 (43\%) & \\
\hline BMI & $24.5 \pm 2.1$ & $26.5 \pm 4.1$ & 0.078 \\
\hline
\end{tabular}

Numerical data are expressed as mean \pm standard deviation; $\mathrm{BMI}=$ body mass index 
TABLE 2. Percentage of tibial tunnel wall cortical bone in both groups at follow-up examinations. Values are given as means $(95 \%$ confidence interval)

\begin{tabular}{cccc}
\hline Period from surgery (months) & $\begin{array}{c}\text { Control group } \\
(\mathrm{n}=\mathbf{2 0})\end{array}$ & $\begin{array}{c}\text { PRP group } \\
(\mathbf{n}=21)\end{array}$ & $\begin{array}{c}\text { p-values for differences } \\
\text { between groups }\end{array}$ \\
\hline 1 & $5.0(1.8-8.2)$ & $6.7(1.8-11.5)$ & 0.928 \\
2.5 & $22.5(17.3-27.7)$ & $36.2(28.7-43.7)$ & 0.004 \\
6 & $53.5(47.0-60.0)$ & $67.1(61.0-73.3)$ & 0.003 \\
\hline
\end{tabular}

values of TCB between the three control examinations with increasing postoperative time $(\mathrm{p}<0.001$ in all paired comparisons).

At the first postoperative month we found only small amount of TCB in each group with a nonsignificant difference between the groups $(\mathrm{p}=0.928)$. At two and a half months as well as at six months after surgery, the mean percentage of TCB (Table 2) was significantly higher in the PRP group than in the control group (36.2 vs 22.5 and 67.1 vs 53.5, p = 0.004 and $\mathrm{p}=0.003$, respectively).

\section{Discussion}

For ethical reasons, histological evaluation of ACL graft incorporation in humans is impossible, particularly in the bone tunnels. However, MRI is a method of choice for the evaluation of the knee. ${ }^{28}$ Graft healing in the tibial tunnel starts immediately after the operation as an acute inflammatory response with oedema, neutrophils and recruited macrophages present in the tendon bone interface as early as 4 days after surgery. ${ }^{2,3}$ At 3 weeks, small vessels appear along with an increased number of osteoblasts on the bone surface. After 6 weeks, the vessels decrease in number and there is a shieldlike new bone formation surrounding the graft as well as an increased number of collagen fibers integrating along the tendon. ${ }^{6}$ Sharpey-like fibers, which anchor the fibroproliferative process to the bone, were found at 6 to 12 weeks after reconstruction in a dog model, followed by progressive bone ingrowth. ${ }^{2,3}$ The tissue maturation process is finished at about 26 weeks, although this differs among various animals and also between different types of grafts. ${ }^{1,2,4}$

In the intratunnel graft healing process, incorporation progresses through the formation of a new matrix at the tendon-bone interface. Proliferation of new bone trabeculae along the edge of the tunnel is seen as early as three weeks after surgery. ${ }^{6}$ This pattern is not uniform, as some areas exhibit a cartilaginous interface between tendon and bone. The zone of fibrocartilage may persist and represents a form of direct healing by tissue which may undergo enchondral bone formation. ${ }^{6}$ This histological evidence is consistent with our observation of focal areas of sclerosis, representing the TCB, which in the follow-up period in each group expanded and finally fused. Although, on average, two thirds of the tibial wall circumference were sclerotic in the PRPG group at six months, we did not detect complete TCB formation surrounding the entire tunnel in any patient. This could be the consequence of a relatively short follow-up period and is in accordance with the observation of increased osteoblastic activity up to two years after reconstruction. ${ }^{4}$

Several reports of the enhancing effect of PDGF and various other GFs on bone proliferation exist, particularly in periodontology. ${ }^{11-25}$ These factors have been shown to enhance murine osteoblast activity and proliferation in vitro. ${ }^{23}$ In vivo, 2-fold increases in uptake of the bone-seeking radiopharmaceutical Technetium 99-MDP, as well as histologically up to a 10-fold increase in new bone and cement were found in dogs. ${ }^{21}$ Locally added PRP accelerated bone healing after mandibular reconstruction in goats considerably. ${ }^{25}$ In humans, significant increase in alveolar bone formation in the process of periodontal regeneration was demonstrated as effects of locally applied PDGF and insulin-like GF. ${ }^{22,24}$

Despite the lack of radiological evidence in humans, assessment with MRI in sagittal and coronal slices demonstrated significantly more periosteal and intratunnel bone formation as effects of locally applied combination of bone GF during the ACL reconstruction in rabbits. This was histologically confirmed with more extensive new bone trabeculae and cartilage formation at the tendon-bone interface at two and eight weeks after reconstruction and generally more mature tissue at the grafttunnel interface. ${ }^{18}$ In addition, other histological studies in animal models also showed more new bone formation at this interface as effects of local application of bone morphogenetic protein (BMP)7 and BMP-2 up to eight weeks after reconstruc- 
tion. ${ }^{19,20}$ Moreover, all of the above-mentioned studies also found higher tensile strength in bone GF groups.

We did not detect differences in the TCB between the groups at one month, which is not in agreement with the above-mentioned animal studies, possibly because of a higher sensitivity with histological analysis, which may detect small changes in the TCB in the first weeks after surgery, when changes are too subtle for observation with MRI. The assessment of joint or even graft stability was not a part of the study protocol which could represent a limitation. However, increased anterior knee stability resulting from PDGF or bone GF has been demonstrated in various studies of humans and animals, ${ }^{18-20,27}$ although the clinical effect of the PRP is still debated. ${ }^{26,29}$ There are several other limitations of the study. We did not evaluate the femoral tunnel because such examination would have to be performed in a different plane, and would therefore significantly prolong the examination. In the tibial tunnel, the analysis of additional neighbouring slices could be more accurate, but also time-consuming, therefore in each patient the same slice was meticulously selected for analysis in all follow-up examinations. Owing to superior spatial resolution with even thinner slices, CT examination could be possibly more accurate, but at the cost of significant radiation exposure, important particularly in this young study population. We did not perform preoperative MRI, nor did we evaluate for lower degrees of osteoarthrosis and concurrent procedures during arthroscopy, as well as injury chronicity and possible drugs intake like NSAR and corticosteroids. All these factors could influence the inflammatory response in the healing process, which is, however, rather questionable in the intratunnel region compared with intra-articular inflammation.

In conclusion, the results of our study demonstrate that formation of focal areas of sclerotic cortical bone with subsequent fusion into a thick tibial tunnel wall is a part of the ACL graft incorporation process that can be quantitatively assessed by MRI. Furthermore, we observed that local application of PRP results in enhanced cortical bone formation encircling the tibial tunnel at 2.5 and 6 months but not 1 month after ACL reconstruction.

\section{References}

1. Arnoczky SP, Tarvin GB, Marshall JL. Anterior cruciate ligament replacement using patellar tendon. An evaluation of graft revascularisation in dog. J Bone Joint Surg Am 1982; 64: 217-24.
2. Rodeo SA, Arnoczky SP, Torzilli PA, Hidaka C, Warren RF. Tendon-healing in a bone tunnel. A biomechanical and histological study in a dog. J Bone Joint Surg Am 1993; 75: 1795-803.

3. Scranton PE Jr, Lanzer WL, Ferguson MS, Kirkman TR, Pflaster DS. Mechanisms of anterior cruciate ligament neovascularisation and ligamentisation. Arthroscopy 1998; 14: 702-16.

4. Goradia VK, Rochat MC, Grana WA, RohrerMD, Prasad HS. Tendon-to-bone healing of a semitendinosus tendon autograft used for $\mathrm{ACL}$ reconstruction in a sheep model. Am J Knee Surg 2000; 13: 143-51.

5. Kawamura S, Ying L, Kim HJ, Dynbyl C, Rodeo SA. Macrophages accumulate in the early phase of tendon-bone healing. J Orthop Res 2005; 23: 1425-32.

6. Deehan DJ, Clawston TE. The biology of integration of the anterior cruciate ligament. J Bone Joint Surg Br 2005; 87: 889-95.

7. Heldin $\mathrm{CH}$, Westermark B. PDGF-like growth factors in autocrine stimulation of growth. J Cell Physiol Suppl 1987; Suppl 5: 31-4.

8. Anitua E, Andia I, Ardanza B, Nurden P, Nurden AT. Autologous platelets as a source of proteins for healing and tissue regeneration. Thromb Haemost 2004; 91: 4-15.

9. Mehta S, Watson JT. Platelet rich concentrate: basic science and current clinical applications. J Orthop Trauma 2008; 22: 432-8.

10. Marx RE. Platelet-rich plasma (PRP): what is PRP and what is not PRP? Implant dent 2001; 10: 225-8.

11. Weiler A, Forster C, Hunt P, Falk R, Jung T, Unterhauser FN, et al. The influence of locally applied platelet-derived growth factor-BB on free tendon graft remodeling after anterior cruciate ligament reconstruction. $\mathrm{Am} J$ Sports Med 2004; 32: 881-91.

12. Yamazaki S, Yasuda K, Tomita F, Tohyama H, Minami A. The effect of transforming growth factor-beta1 on intraosseous healing of flexor tendon autograft replacement of anterior cruciate ligament in dogs. Arthroscopy 2005; 21: 1034-41.

13. Yoshikawa $T$, Tohyama $H$, Katsura $T$, Kondo $E$, Kotani $Y$, Matsumoto $H$, et al. Effects of local administration of vascular endothelial growth factor on mechanical characteristics of the semitendinosus tendon graft after anterior cruciate ligament reconstruction in sheep. Am J Sports Med 2006; 34: 1918-25.

14. Nagumo A, Yasuda K, Numazaki H, Azuma H, Tanabe Y, Kikuchi S, et al Effects of separate application of three growth factors (TGF-beta1, EGF and PDGF-BB) on biomechanical properties of the in situ frozen-thawed anterior cruciate ligament. Clin Biomech (Bristol, Avon) 2005; 20: 283-90.

15. Xu J, Shen J, Ding Y, Shen HY, Zeng ZP, Ma RF, et al. The clinical value of combined use of MR imaging and multi-slice spiral $\mathrm{CT}$ in limb salvage surgery for orthopaedic oncology patients: initial experience in nine patients. Radiol Oncol 2012; 46: 189-97.

16. Wang $\mathrm{X}, \mathrm{Xu} \mathrm{M}$, Liang $\mathrm{H}, \mathrm{Xu}$ L. Comparison of $\mathrm{CT}$ and MRI in diagnosis of cerebrospinal leak induced by multiple fractures of skull base. Radiol Oncol 2011; 45: 91-6.

17. Robinson J, Huber C, Jaraj P, Colombet P, Allard M, Meyer P. Reduced bone tunnel enlargement post hamstring $\mathrm{ACL}$ reconstruction with poly-L-lactic acid/hydroxyapatite bioabsorbable screws. Knee 2006; 13: 127-31.

18. Anderson K, Seneviratne AM, Izawa K, Atkonson BL, Potter HG, Rodeo SA. Augmentation od tendon healing in an intraarticular bone tunnel with use of a bone growth factor. Am J Sports Med 2001; 29: 689-98.

19. Mihelič R, Pecina $M$, Jelic $M$, Zoricic $S$, Kusec V, Simic $P$, et al. Bone morphogenetic protein-7 (osteogenic protein-1) promotes tendon graft integration in anterior cruciate ligament reconstruction in sheep. Am J Sports Med 2004; 32: 1619-25.

20. Rodeo SA, Suzuki K, Deng XH, Wozney J, Warren RF. Use of recombinant human bone morphogenetic protein-2 to enhance tendon healing in a bone tunnel. Am J Sports Med 1999; 27: 476-88.

21. Lynch SE, de Castilla GR, Williams RC, Kiritsy CP, Howell TH, Reddy MS, et al The effects of short-term application of a combination of platelet-derived and insulin-like growth factors on periodontal wound healing. I Periodontol 1991; 62: 458-67.

22. Howell TH, Fiorellini JP, Paquette DW, Offenbacher S, Giannobile WV, Lynch SE. A phase I/II clinical trial to evaluate a combination of recombinant human platelet-derived growth factor-BB and recombinant human insulin-like growth factor-I in patients with periodontal disease. J Periodontol 1997; 68: 1186-93. 
23. Mott DA, Mailhot J, Cuenin MF, Sharawy M, Borke J. Enhancement of osteoblast proliferation in vitro by selective enrichment of demineralized freeze-dried bone allograft with specific growth factors. J Oral Implantol 2002; 28: 57-66.

24. Nevins M, Camelo M, Nevins ML, Schenk RK, Lynch SE. Periodontal regeneration in humans using recombinant human platelet-derived growth factor-BB (rhPDGF-BB) and allogenic bone. J Periodontol 2003; 74: 1282-92.

25. Fennis JP, Stoelinga PJ, Jansen JA. Mandibular reconstruction: a histological and histomorphometric study of the use of autogenous scaffolds, particulate cortico-cancelous bone grafts and platelet rich plasma in goats. Int Oral Maxillofac Surg 2004; 33: 48-55.

26. Nin JR, Gasque GM, Azcárate AV, Beola JD, Gonzales MH. Has platelet-rich plasma any role in anterior cruciate ligament allograft healing? Arthroscopy 2009; 25: 1206-13.

27. Vogrin $M$, Rupreht $M$, Dinevski D, Hašpl $M$, Kuhta $M$, Jevsek $M$, et al. Effects ofa platelet gel on early graft revascularization after anterior cruciate ligament reconstruction: a prospective, randomized, double-blind, clinical trial. Eur Surg Res 2010; 45: 77-85.

28. Vasilevska V, Szeimies U, Staebler A. MRI diagnostis of Baker cyst and significance of associated medial compartment knee osteoarthritis. Radiol Oncol 2008; 42: 51-8.

29. Vavken P, Sadoghi P, Murray MM. The effect of platelet concentrates on graft maturation and graft-bone interface healing in anterior cruciate ligament reconstruction in human patients: a systematic review of controlled trials. Arthroscopy 2011; 27: 1573-83. 